\title{
The development of a new method for making justified decisions by municipal authorities in the management of territories on the basis of the results of the environmental express-control of the state of various media
}

\author{
Egor Rukin ${ }^{1 *}$, Angelina Moroz ${ }^{1}$, Konstantin Smirnov ${ }^{2,3}$, Vadim Davydov ${ }^{1,4}$, and Victoria \\ Yushkova ${ }^{5}$ \\ ${ }^{1}$ Peter the Great Saint Petersburg Polytechnic University, Higher School of applied physics and space \\ technologies, Polytechniheskya 29, Saint-Petersburg, 195251, Russian Federation \\ ${ }^{2}$ OJSC "NRI "Electron", sector of photonics, Thorez Avenue 68, Saint-Petersburg, 194223, Russian \\ Federation \\ ${ }^{3}$ The Bonch-Bruevich Saint Petersburg State University of Telecommunications, department of \\ photonics, Bolshevik Avenue 22, Saint-Petersburg, 193232, Russian Federation \\ ${ }^{4}$ All Russian Research Institute of Phytopathology, sector of biology, Big Vyazem 5, Moscow Region, \\ 143050, Russian Federation \\ ${ }^{5}$ Saint-Petersburg University of Management Technologies and Economics, Lermontovsky Avenue 44, \\ Saint-Petersburg, 190109, Russian Federation
}

\begin{abstract}
The article considers the problems of assessing the ecological state of the water body and the surrounding area in the municipalities of St. Petersburg. The necessity of using the methods of express-control to determine the state of different media to obtain reliable operational information. A new method of making an informed decision on the territory management based on the results of express-control is proposed. The results of the study of the water body and adjacent areas are presented.
\end{abstract}

\section{Introduction}

In a fairly difficult environmental situation, especially in large cities such as St. Petersburg, the government in oreder to solve the problem of environmental control actively involves local authorities (municipalities) [1-4]. This is due to the different types of pollution and sources of them creation (for example, industrial and transport enterprises, building objects, etc.) [5-12]. It is very difficult to quickly monitor the ecological situation [13-15]. The experience of municipalities in Europe has shown the effectiveness of this approach. In most cases, due to the large differences in management systems, as well as in the legal framework between the countries of Europe and Russia, the methods used in European countries, fail in their use in the Russian Federation. Therefore, taking into account the experience of European countries, it is necessary for the Russian Federation to develop its own methods.

* Corresponding author: rukin.egor@gmail.com 
In these methods it is necessary to consider the specificity of the territorial structure of local self-government bodies - municipalities. In different cities and towns, municipalities have different authorities and they solve different tasks. One of the common tasks that all municipalities have to solve is to control the state of the environment and take measures to prevent pollution of the territory. This requires the use of various instruments, methods and technologies [14-23]. In addition, municipalities organize work to clean the area from pollution and stop the activities of organizations and entrepreneurs polluting the territory, they also impose fines for violation of the ecological state of the territory.

Municipalities in large cities (for example, Moscow, St. Petersburg, etc.) have the greatest difficulties in solving the problems of environmental control of the territory and making decisions on its management. In these cities, municipalities have problems very similar to those that are present in European countries, only they have Russian specifics. Methods for their solution are interesting not only for Russian municipalities, but for European ones, as population migration takes place. Currently in Europe there are many citizens from Russia who are engaged in business.

The greatest difficulties in the work of public authorities arise when monitoring the state of medium and small water bodies on the territory of St. Petersburg, as well as their coastal areas. For other large cities of Russia, the situation under consideration in most cases has insignificant differences. Therefore, the proposed solutions in this work will be relevant for other large cities of Russia.

\section{The problems and method for solving them}

Medium and small water bodies in large cities include lakes, ponds in parks and recreation areas, as well as small and medium-sized rivers and streams (for example, in St. Petersburg - Melnichnyy Ruchey, which can be compared to a small river) [8-10]. In most cases, these rivers and streams flow into a large river that flows through the city, or into the gulf (for example, Gulf of Finland) on the coast of which the city is located, rarely directly into the sea. The problem with monitoring the status of lakes, ponds and ponds in parks and recreation areas, as well as their coastal areas is solved to a certain extent, whereas there are great difficulties with such problems for small rivers and streams [8-10, 14, 15, 20]. Consider the example of the Krasnenkaya river, which flows through the territory of five municipalities. Statistical studies have shown that in St. Petersburg there are more than 60 municipalities out of 111 with small rivers and streams. Therefore, we can assume that the problem under consideration and its proposed solutions are relevant for the whole city. This is a very unfavorable from the point of view of ecology water body, flowing into the waters of the Gulf of Finland.

The Kranenkaya river passes through a variety of industrial and commercial zones. In industrial zones there are medium and small businesses engaged in road transport, repair and maintenance services associated with increased environmental risk (for example, the dismantling of used batteries, etc.). These organizations do not have large working capital, so they have to minimize their costs by all means. Many years of our research, as well as other scientists, show that the first thing they have to save on is ecology $[1,2,4,6,8,16,17$, 23]. Various wastes containing dangerous substances has been continiously disposed into the Krasnenkaya river and its coastal area. This led to the fact that the water in the Krasnenkaya river became of muddy color with a specific smell. The presence of such a smell means that there is a high carbon exchange in the river, which destroys many river inhabitants. Our measurements have confirmed the presence of a large number of dangerous carbon compounds in the river.

It should be noted that in most places of the waterway of the Krasnenkaya river access to its coastal regions is limited for various reasons (fig. 1). 


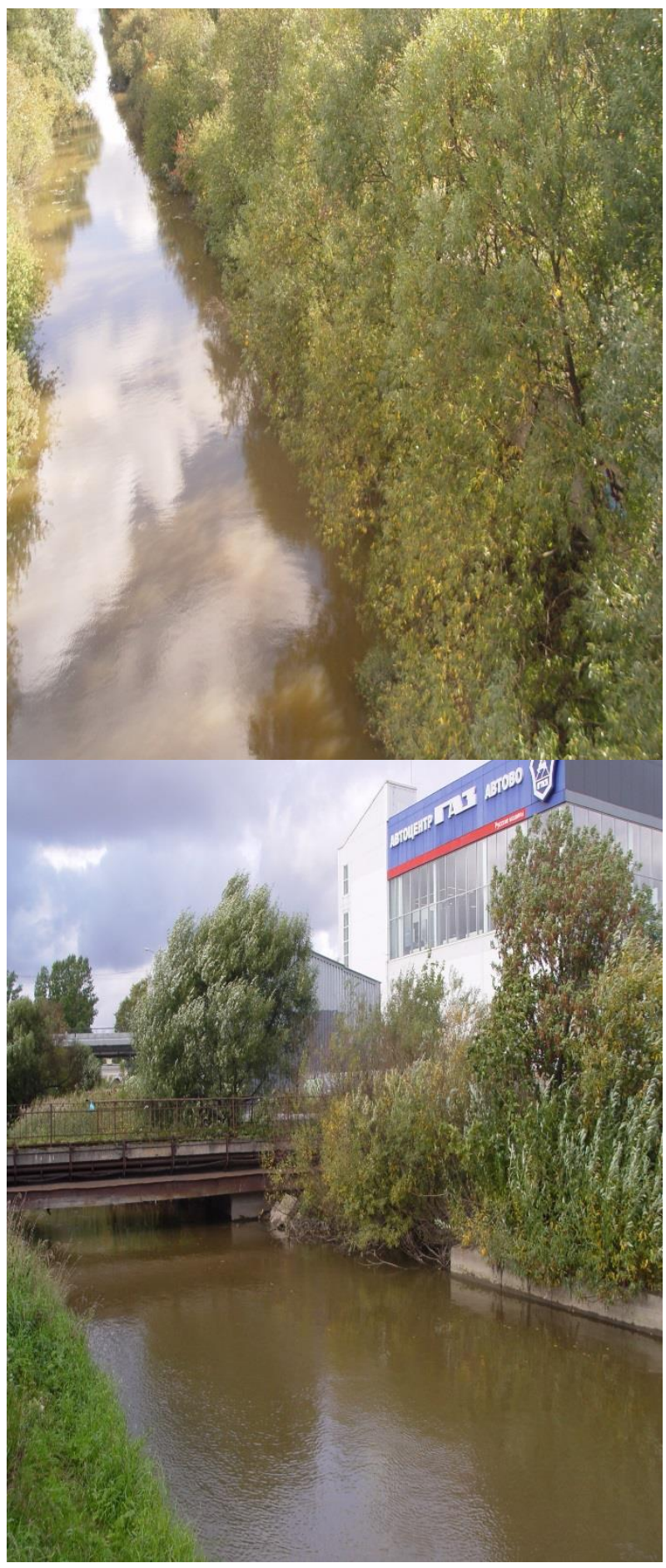

Fig. 1. The appearance of the waterway and coastal areas of the river. 
In order to go to its coast and take samples of water and soil, it is necessary to know the specifics of the territory of the object. As our practical work has shown, this can only be carried out by the employees of the municipality, as they are sitatuated closely to these territories and familiar with the real situation. Previously carried out measures to control the state of the water in the Krasnenkaya river in the port, where it flows, were found to be ineffective. Since public services stated the fact of pollution and urgently carried out work to eliminate it. The reason of pollution and guilt was impossible to establish. This impunity has led to an increase in pollution in the river.

To carry out regular monitoring (several times a day) of the state of the water and the coastal zone at this fairly long water body, various public services do not have opportunities for various reasons. The most optimal decision was to carry out operational monitoring of the state of water in the river and soil on the coast by municipal employees, who can do it several times a day.

Long experience in monitoring the state of water and the coastal zone of these water bodies has shown that for the efficient work it is necessary to use express-methods $[3,5,8$, 11, 13, 14, 18, 21-23]. These methods are mainly implemented in small devices. Municipalities do not have chemical laboratories. It is desirable to carry the collected samples to stationary laboratories for analysis only in the case of significant deviations from the standard state detected $[17,20,21,23]$. In these cases, a specialized service is also called to the place of the identified source of pollution, and it is desirable to send primary information about the source of pollution for more effective work prior to the call.

If the approximate composition of the substance that caused the primary contamination is known and the exchange of information between different services is established, it is highly likely that the source of the contamination and the culprit will be identified. Traces of dumping indicating the location, depending on weather conditions, remain at least for 2-3 hours. Employees of five municipalities very quickly go to the territory of the river, which is located in most cases in walking distance, and do the necessary activities.

In addition, in case of an emergency, it is possible to quickly ban access to the water body, warn all services of the seaport of the approaching pollution of its waters, etc. In this case, the economic losses from the effects of pollution would be several times less.

Our studies have shown that the successful implementation of this technique is possible only when using the methods of express-control, as the large number of different samples that one have to take. In addition, the time for analysis of each sample should not exceed a few seconds. In this case, chemical express methods were ineffective $[8,12,14,15]$. It is necessary to carry a large number of hazardous reagents and specialized glass cuvettes. In conditions when it is necessary to move on the territory of the object from 2 to $3 \mathrm{~km}$, measuring devices should be easy to move and provide people with the necessary data to assess the environmental condition of a given area.

Taking into account the nature of pollution in small urban rivers, a method of using three small measuring devices to assess the environmental situation, conducting measurements in express mode was proposed.

The first one is a multifunctional dosimeter that detects the levels of three types of radioactive radiation $(\alpha, \beta$ and $\gamma)$ with autonomous power supply. The second is an optical concentrator for determining the content of petroleum products in water (fig. 2.a), the third developed by us small-sized NMR spectrometer (fig. 2.b, c) operating on the nuclear magnetic resonance (NMR) phenomenon $[17,19]$. 

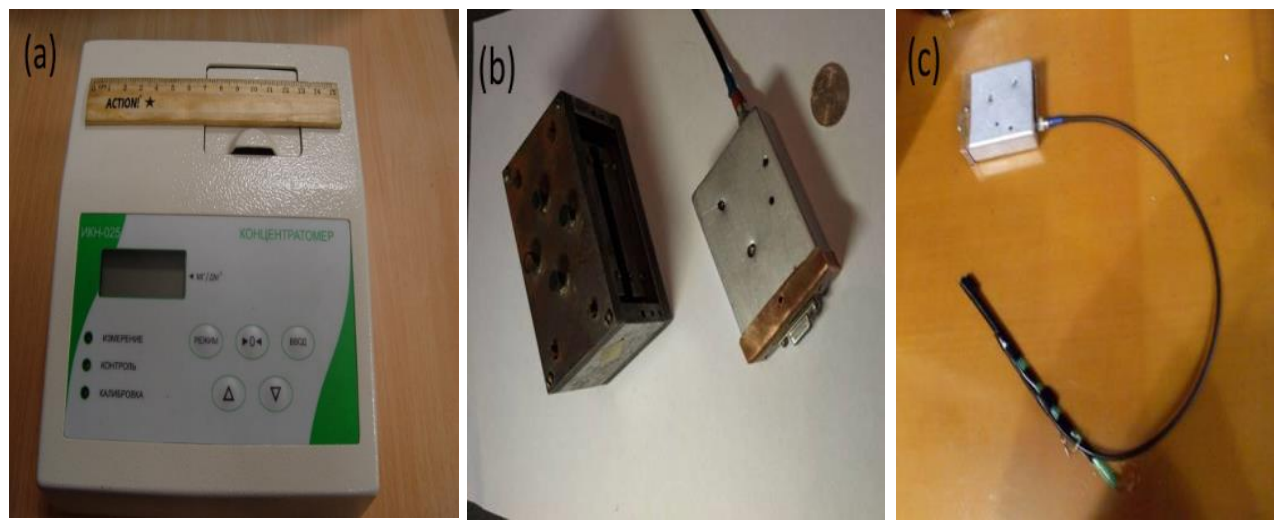

Fig. 2 (a, b, c). The compact devices for express-control: (a) - optical concentrator, (b) - compact magnetic system and NMR signal detection device, (c) - NMR signal detection device with measuring probe.

Small-sized concentrator and NMR spectrometer also hasve autonomous power sources. In addition, data received from them immediately goes to the input of a laptop or tablet. Monitoring of the water body and its coastal area is carried out with reference to the coordinates in real time. The tablet allows you to use GPS navigation.

The use of these devices allows you to quickly move along the coastal zone of the river and take samples in a large number of places, since the small weight of these devices allows you to use a large number of containers. Studies have shown that this technique allows to identify in real time the source of pollution, in some cases not a single one. Quickly transmit information on pollution to other services, etc. In addition, only samples where contamination was detected are sent to the stationary laboratory for additional studies, which are quite expensive. This saves time and financial resources of the municipality.

In case of an emergency, the municipality may close access to the site and take action before the arrival of the Ministry of emergency situations to prevent further contamination.

The use of express methods, as shown by the practice of their application, allows carrying out this control of the territory of the water body more than once a day, which disciplines businessmen working in the area.

\section{Results of experimental investigations and discussion}

On the figure 3 shows, as an example, the results of a study of the oil content in the water (concentration $\mathrm{N}_{\text {o.p. }}$ ) along the river channel $(20 \mathrm{~cm}$ from the shore) during the day after the detection of pollution. 


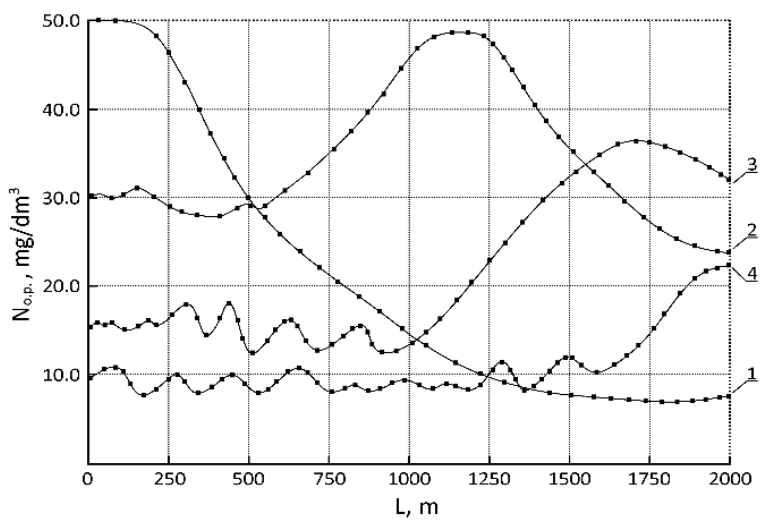

Fig. 3. The concentration $N_{\text {o.p. }}$ change on length $\mathrm{L}$ of river channel. The value $\mathrm{L}$ is measured in the direction of the river flow. Graphics 1,2, and 4 correspond to the following time intervals in the hours in which measurements were taken: from 8 to 10 ; from 10 to 12 ; from 12 to 14 ; from 14 to 16 .

Figure 4 shows, as an example, the values of longitudinal $\mathrm{T}_{1}$ and transversal $\mathrm{T}_{2}$ of water relaxation time $[13,14,19]$ at the same points and in the corresponding time intervals are presented.
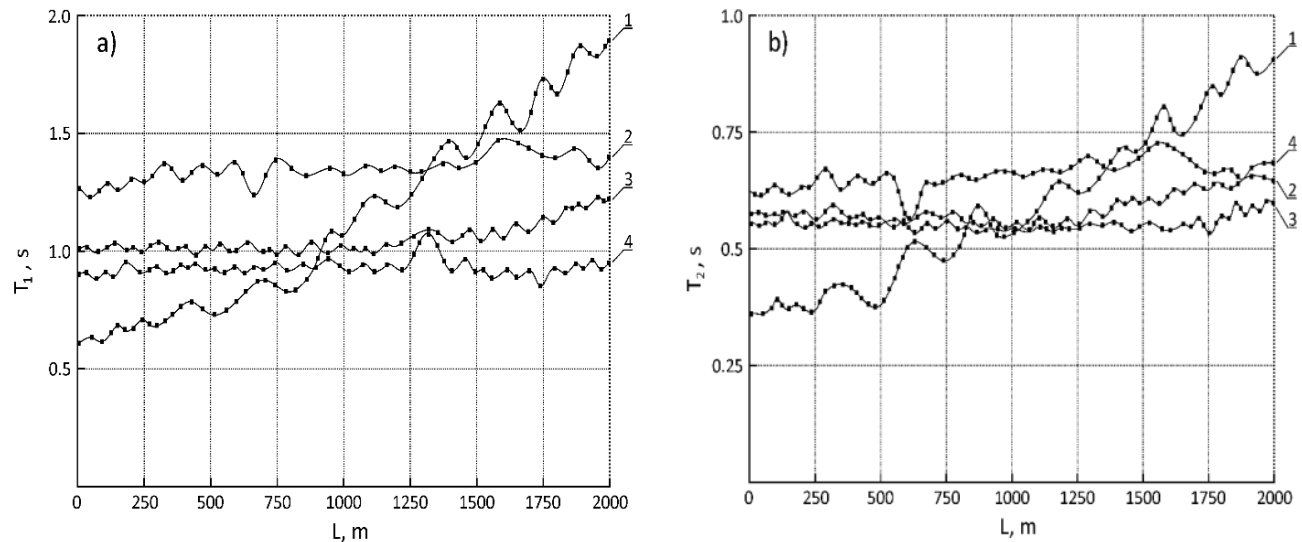

Fig. 4 (a. b). The change of relaxation time values on length $L$ of river channel: (a) $-T_{1} ;(\mathbf{b})-T_{2}$. The value $\mathrm{L}$ is measured in the direction of the river flow. Graphics 1, 2, and 4 correspond to the following time intervals in the hours in which measurements were taken: from 8 to 10 ; from 10 to 12 ; from 12 to 14 ; from 14 to 16 .

The analysis of the results shows that the level of oil products in the water in the primary source of pollution after 8 hours reached the average statistical norm for the Krasnenkaya river. This shows that it will be extremely difficult to identify the source of pollution after this time period.

In some places of the waterway there is a sharp change in the concentration of petroleum products (decrease more than the measurement error), the measured values of $T_{1}$ and $T_{2}$ also confirm the presence of this process. The reason for this change may be different, for example, the appearance of other substances that react chemically with petroleum products. Additional research and inspection of the area is required.

If we consider the case presented in figs 3 and 4, the results of additional studies on the river bed have shown that there were found expired packed bags of phosphate fertilizers. The packaging deteriorated over time and passed a slow flow of fertilizers with a high 
concentration of phosphorus into the water. The most dangerous source of pollution for living organisms was removed from the river.

The total practical implementation of the developed method and the decisions taken on the basis of the data obtained with the help of it is shown in fig. 5. As an example, the data on the change in the concentration level $\left(\mathrm{N}_{\mathrm{o}}\right)$ of oil products in the Krasnenkaya river for spring-summer-autumn 2017 are given.

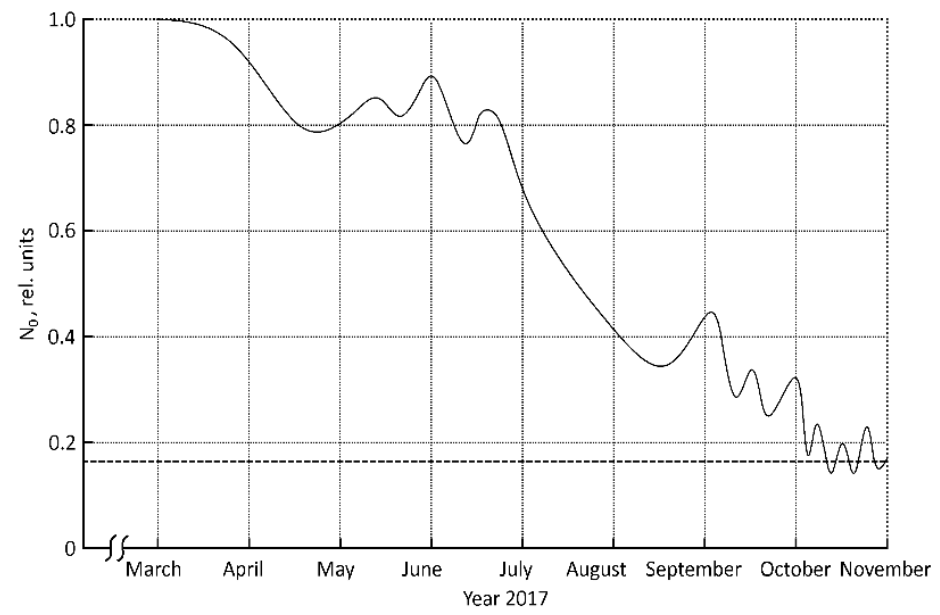

Fig. 5. The change of concentration level $\mathrm{N}_{0}$ in following time intervals from March to November 2017.

These substances, as shown by studies, pollute the water and the coastal area of the river the most. The results show a fivefold decrease in the concentration of petroleum products by November 2017. For the mid-December average level of oil products concentration in the water of the river Krasnenkaya established at the level of $5.5 \mathrm{mg} / \mathrm{dm}^{3}$. It should be noted that the average level of oil in natural waters in the Leningrad region varies from 0.5 to 1.5 $\mathrm{mg} / \mathrm{dm}^{3}$.

In addition, the use of the developed small-sized NMR spectrometer makes it possible to determine the concentration of some hazardous chemical elements in water in the expressmode (for example, lithium, phosphorus, sodium, etc.), if their content is sufficient to register the NMR signal $[8,14,19]$. In the case of a significant excess of the maximum permissible concentration, for example, for lithium $2 \mathrm{mg} / \mathrm{dm}^{3}$, no problem will occur with the registration of the NMR signal.

\section{Conclusion}

The results of the monitoring of the state of water and the coastal area of the river Krasnenkaya showed the effectiveness of our method for monitoring the environmental situation, as well as on the basis of the results of environmental monitoring of the territory to make informed decisions by various authorities, especially municipalities.

Unfortunately, in addition to unauthorized dumpings of wastewater and chemicals into the Krasnenkaya river, violations of the sewerage system at many facilities were found, the pipes are old and damaged. Untreated water flows through the ground into the river Krasnenkaya. Restoration of the sewerage system is very difficult and time-consuming work. For this reason, the reduction of pollution in the river Krasnenkaya in 2018 almost stopped. 
A new technique for the determination of sewerage systems damages based on water and soil express-monitoring is being developed.

In the future, it is planned to improve the developed method of monitoring the ecological state of water and the coast of water bodies in order to optimize the work with the various services of the city to improve the ecological state of the Krasnenkaya river, which automatically affects the ecology of the Gulf of Finland.

\section{References}

1. D. Nemova, R. Bagautdinov, A. Mushnikov, Procedia Engineering, 117(1) 1120-1126 (2015)

2. V. Mushchanov, V. Sievka, A. Veshnevska, D. Nemova, Procedia Engineering, 117(1) 1018-1026 (2015)

3. V. V. Davydov, V. I. Dudkin, A. Yu. Karseev, V. A. Vologdin, Journal of Applied Spectroscopy, 82(6) 1013-1019 (2016)

4. R. V. Davydov, V. I. Antonov, D. V. Molodtsov, A. Trebukin, Advances in Intelligent Systems and Computing, 692 915-920 (2018)

5. M. A. Baranov, E. N. Velichko, E. T. Aksenov, Journal of Physics: Conference Series, 917(6) 062059 (2017)

6. D. Tarasova, A. Staritcyna, D. Nemova, K. Andreev, MATEC Web of Conferences, $\mathbf{5 3}$ 01007 (2016)

7. N. Vatin, D. Nemova, L. Khazieva, D. Chernik, Applied Mechanics and Materials, 635637 2057-2062 (2014)

8. V. V. Davydov, N. S. Myazin, T. I. Davydova, Russian Journal of Nondestructive Testing, 53(7) 520-529 (2017)

9. S. Bezborodov, N. Zemlynaya, Applied Mechanics and Materials, 725-726 1350-1356 (2015)

10. E. Simonenko, V. Starkov, L. Molodkina, A. Chusov, Applied Mechanics and Materials, 725-726 1357-1362 (2015)

11. Yu. I. Neronov, Measurement Techniques, 60(1) 96-102 (2017)

12. Yu. I. Neronov, N. N. Seregin, Measurement Techniques, 55(11) 1287-1293 (2013)

13. N. S. Myazin, V. V. Davydov, V. V. Yushkova, T. I. Davydova, V. Yu. Rud', Journal of Physics: Conference Series, 917(4) 042017 (2017)

14. N. S. Myazin, S. E. Logunov, V. V. Davydov, V. Yu. Rud', N. M. Grebenikova, V. V. Yushkova, Journal of Physics: Conference Series, 929(1) 012064 (2017)

15. J. Vervoort, J. A. van Leerdam, G. Stroomberg, P. de Voogt, Environmental Science \& Technology, 48 (21) 12791-12799 (2014)

16. V. Maslak, N. Nasonkina, V. Sakhnoskaya, S. Antonenko, D. Nemova, Procedia Engineering, 117(1) 985-994 (2015)

17. V. V. Davydov, V. I. Dudkin, N. S. Myazin, V. Yu. Rud', Instruments and Experimental Techniques, 61(1)140-147 (2018)

18. S. V. Dyachenko, M. A. Vaseshenkova, K. D. Martison, I. A. Cherepkova, A.I. Zhernovoi, Russian Journal of Applied Chemistry, 89(5) 690-696 (2016)

19. V. V. Davydov, N.S. Myazin, Measurement Techniques, 60(2) 183-189 (2017)

20. T. Polivanova, S. Polivanova, N. Kobelev, A. Akul'shin, V. Kobelev, Applied Mechanics and Materials, 725-726 1332-1337 (2015)

21. V. V. Arkhipov, Instruments and Experimental Techniques, 55 692-695 (2012).

22. E. K. Nepomnyashchaya, E. A. Savchenko, E. N. Velichko, E. T. Bogomaz, Journal of Physics: Conference Series, 956(1) 012009 (2017)

23. N. M. Grebenikova, K. J. Smirnov, V. V. Artemiev, V. V. Davydov, S. V. Kruzhalov, Journal of Physics: Conference Series, 1038 (1) 012089 (2018) 